\title{
Como a Monitorização Contínua de Glicose Subcutânea Pode Colaborar na Interpretação dos Valores da HbAlc no Diabetes Melito Tipo 1?
}

\begin{abstract}
RESUMO
O objetivo desta revisão é avaliar como o sistema de monitorização contínua de glicose subcutânea (CGMS ${ }^{\circledR}$ ) pode colaborar na interpretação dos valores da hemoglobina glicada (A1C) no diabetes melito tipo 1. Foi realizada uma revisão bibliográfica dos benefícios, dos métodos, da interpretação e da experiência com $\mathrm{CGMS}^{\circledast}$. A utilização de sensores contínuos pode ser útil na avaliação de pacientes com diabetes melito com oscilações glicêmicas, hipoglicemias graves, especialmente as noturnas, e hiperglicemias pós-prandiais. A identificação de padrões glicêmicos alterados permite ajustes terapêuticos e melhora do controle metabólico. $\mathrm{O} \mathrm{CGMS}^{\circledR}$ é um procedimento de fácil utilização, com efeitos adversos de pequena intensidade e boa tolerância pelos pacientes. Embora o fabricante preconize um período de utilização de até três dias, observa-se que o prolongamento do teste por mais dias permite a obtenção de dados que auxiliam em mudanças terapêuticas superiores às realizadas com os registros do tempo convencional. (Arq Bras Endocrinol Metab 2008;52/2:299-306)
\end{abstract}

Descritores: $\mathrm{CGMS}^{\oplus}$; DM1; HbA1c; Glicemia

\begin{abstract}
How the Continuous Glucose Monitoring System can contribute to HbA1c Interpretation in Type 1 Diabetes Mellitus?

The aim of the present study is a literature revision of the contribution of continuous glucose monitoring system (CGMS ${ }^{\circledR}$ ) in the interpretation of HbA1c levels in type 1 diabetes (T1DM). We performed a revision of the efficacy, benefits, interpretation of methods and experience with this system. The $\mathrm{CGMS}^{\oplus}$ could be useful in T1DM patients that present great glycemic variability or severe hypoglycemia (mainly nocturnal) and postprandial hyperglycemia. The identification of altered patterns or the glycemic variability allows therapeutic adjustment and improvement of the metabolic control. CGMS ${ }^{\circledR}$ is a safe method with a rare occurrence of side effects and it is well-tolerated by patients. The extension of the procedure for more days, over the $72 \mathrm{~h}$ period recommended by the standard test, allows us to get important data to changes and adjustments on the insulin management of the type 1 diabetes mellitus patients. (Arq Bras Endocrinol Metab 2008;52/2:299-306)
\end{abstract}

Keywords: Type 1 diabetes; Glycemia; CGMS; HbA1c

\section{INTRODUÇÃo}

S BENEFícios ALCANÇAdos em longo prazo com o controle metabólico rigoroso na prevenção e na redução das complicações crônicas micro e revisão

\author{
Marcia K. C. Puñales \\ César Geremia \\ Paula Mondadori \\ MARILISA PICKLER \\ ADRIANA FORNARI \\ BALDUINO TSCHIEDEL
}

Instituto da Criança com

Diabetes, Hospital da Criança

Conceição, Grupo Hospitalar

Conceição, Ministério da Saúde,

Porto Alegre, RS, Brasil.

Recebido em 10/12/2007

Aceito em 18/12/2007 
macrovasculares foram fortemente estabelecidos tanto no DM tipo 1 (DMl) $(1,2)$ quanto no DM tipo 2 (DM2) (3). O objetivo do tratamento intensivo do diabetes melito $(\mathrm{DM})$ visa mimetizar o perfil fisiológico da secreção de insulina, com manutenção dos níveis glicêmicos próximos da normalidade, e obtenção dos níveis de hemoglobina glicada Alc em aproximadamente 7\%, sem aumentar a incidência de episódios de hipoglicemias e evitando ao máximo a ampla variabilidade glicêmica (4-6). A American Diabetes Association (ADA) recomenda níveis de glicemias de jejum e préprandiais entre $90 \mathrm{mg} / \mathrm{Dl}$ e $130 \mathrm{mg} / \mathrm{dL}$, duas horas pós-prandiais < $180 \mathrm{mg} / \mathrm{dL}$ e Alc até um ponto percentual acima do método do laboratório $(<7,0 \%)(6)$. Entretanto, a Associação Americana de Endocrinologia (ACE) e a Federação Internacional de Diabetes (IDF) sugerem que os níveis de Alc devam ser mantidos em níveis ainda mais baixos $(<6,5 \%)(7,8)$. Em crianças e adolescentes (< 13 anos), idosos ( $>65$ anos), indivíduos com história de hipoglicemias despercebidas, comorbidades ou doenças macrovasculares graves, os alvos glicêmicos recomendados pela ADA podem ser mais flexíveis, sendo aceitável manter as glicemias de jejum e préprandiais entre 80 e $160 \mathrm{mg} / \mathrm{dL}$, duas horas pós-prandiais em até $200 \mathrm{mg} / \mathrm{dL}$, e níveis de Alc em até dois pontos percentuais acima do método (6). Os níveis de Alc $<7,5 \%$, recomendados em crianças e adolescentes, deveriam ser obtidos sem o aumento dos episódios de hipoglicemia (9-12).

O tratamento intensivo (múltiplas doses de insulina) e a infusão subcutânea contínua de insulina (CSII), associados à automonitorização freqüente da glicemia capilar, têm mostrado ser efetivos na melhora do controle metabólico (13). Pacientes em tratamento intensivo durante o estudo The Diabetes Control and Complications Trial (DCCT) apresentaram incidência 3,3 vezes mais elevada de hipoglicemia grave a despeito da realização da automonitorização da glicose por quatro ou mais vezes ao dia (1). Existem evidências também de que a melhora na hiperglicemia pós-prandial e a redução na variabilidade glicêmica, não detectadas satisfatoriamente pela $\mathrm{Alc}$, possam ser importantes na diminuição das complicações crônicas do diabetes. Assim, o objetivo do tratamento intensivo é normalizar a Alc, controlar as excursões glicêmicas pós e inter-prandiais e limitar o número e a gravidade dos eventos hipoglicêmicos (14).

Entre as dificuldades para se obter melhor controle metabólico, está a medida intermitente das glicemias, já que a automonitorização pela glicemia capilar (GC) fornece apenas dados intermitentes do controle glicêmico, impedindo uma visão mais ampla do perfil glicêmico do indivíduo ao longo do dia e não fornecendo informações suficientes das variações glicêmicas pós e inter-prandiais e/ou noturnas e detecção das hipoglicemias assintomáticas (15-18).

Entretanto, há alguns anos tornou-se possível e disponível a monitorização contínua de glicose, permitindo aferições da concentração da glicose intersticial e apresentando boa correlação com a glicemia plasmática (19-24). Essa monitorização possibilita avaliar com exatidão as excursões da glicemia ao longo do dia, permitindo otimização do tratamento e melhora do controle metabólico, com redução dos riscos associados à terapêutica insulínica, como as hipoglicemias graves, especialmente as noturnas, tanto no DMl quanto no DM2 (23,25-28).

No Brasil, o sistema de monitorização contínua de glicose $\left(\mathrm{CGMS}^{\circledast}\right)$ é o único sensor disponível comercialmente. A glicose é mensurada com base na reação eletroquímica da enzima glicose oxidase, presente no sensor, com a glicose do fluido intersticial. Os limites de detecção variam de 40 a $400 \mathrm{mg} / \mathrm{dL}$ e são captados a cada dez segundos. A média desses valores é registrada a cada cinco minutos, no total de 288 medidas ao dia e 864 medidas durante o exame preconizado de 72 horas. O sistema é capaz de armazenar até 14 dias de dados contínuos. O aparelho pesa 114 gramas e tem as dimensões de um pager $(9,0 \times 7,0 \times 2,2 \mathrm{~cm})$. É capaz de operar em temperatura ambiente que varia de 0 a 50 graus centígrados. Podem-se registrar diversos eventos, como refeições, exercício e doses de insulina para facilitar as correlações que serão feitas posteriormente. Para calibrar o monitor, os pacientes devem inserir quatro medidas de glicemia capilar a cada dia. Ao final do registro, o sensor é descartado, e os dados armazenados são transferidos a um software (Solutions Software) para análise. Esse programa é capaz de gerar diversos formatos de gráficos, tabelas e fluxogramas de fácil interpretação e demonstração para o paciente.

Embora o fabricante preconize um período de medição de três dias, é possível o prolongamento do exame por até cinco a sete dias, o que possibilita uma análise preliminar dos dados no terceiro dia, ajuste na terapêuti$\mathrm{ca}$, e dois a quatro dias adicionais para se aferirem os resultados. É importante efetuar e incluir um mínimo de quatro valores de GC por dia, para que o sensor possa correlacioná-los aos da glicemia intersticial. 
Uma das limitações bem conhecidas do $\mathrm{CGMS}^{\circledR}$ é a elevada taxa de detecção de hipoglicemias assintomáticas, principalmente as noturnas, muitas das quais podem ser espúrias, podendo ser minimizadas com a calibração freqüente por intermédio da inserção de glicemias capilares no sistema (13). Todavia, a eficácia do $\mathrm{CGMS}^{\circledR}$ na identificação de hipoglicemia noturna assintomática e seu impacto sobre o controle metabólico a longo prazo foram comparados em 70 pacientes com $\mathrm{DM}(\mathrm{DMl}=40 ; \mathrm{DM} 2=30)$ em relação à monitorização pela GC (23). Os resultados demonstraram que o $\mathrm{CGMS}^{\circledR}$ foi capaz de detectar hipoglicemias assintomáticas em $62,5 \%$ dos indivíduos com DMl e 46,6\% com DM2; 73,7\% dos eventos ocorreram no período noturno (23). Os autores sugerem que o CGMS ${ }^{\circledR}$ mostra-se superior à monitorização intensiva pela $\mathrm{GC}(8 \mathrm{x} / \mathrm{dia})$ na identificação de hipoglicemia assintomática, inclusive no período noturno. Nesse estudo, porém, não houve redução de Alc ou melhora do controle glicêmico quando comparada à monitorização intensiva $(8 \mathrm{x} / \mathrm{dia})$ pela GC (23).

Diferentes estudos têm demonstrado a eficácia da monitorização contínua da glicose na redução da $\mathrm{Alc}$ $(29,30)$. Em estudo com 12 adolescentes com DMl e Alc $>8 \%$, que realizaram $\mathrm{CGMS}^{\circledR}$ por período de 72 horas, excursões glicêmicas não detectadas pela GC foram detectadas pelo sensor em $100 \%$ dos casos, episódios de hiperglicemia pós-prandial foram identificados em 83,3\% e de hipoglicemia noturna em 33\%, com diminuição significativa das oscilações na glicemia e nos níveis da Alc $(\mathrm{p}<0,05)$ após dois meses do exame (30). Os resultados obtidos com o uso do CGMS $^{\circledR}$, por período de 72 horas, em 91 crianças com DMI, e excursões glicêmicas induzidas, demonstraram maior acurácia para níveis mais elevados de glicose e menor sensibilidade para episódios de hipoglicemia ( $\mathrm{p}$ $<0,05$ ) (27). Outro estudo evidenciou que o uso do CGMS $^{\circledR}$ em 72 horas apresenta boa acurácia e efetividade na detecção de episódios de hiperglicemia pósprandial $(90 \%)$ e de hipoglicemia assintomática $(70 \%)$, sendo um método de avaliação de grande importância para otimização do controle metabólico de crianças com DMl (31) e com sensibilidade de $33 \%$ para detecção de hipoglicemias (32). Alguns estudos, todavia, relatam menor eficácia e acurácia na detecção de níveis glicêmicos reduzidos ou episódios hipoglicêmicos assintomáticos e referem que a principal indicação do CGMS $^{\circledast}$ está na melhora do controle metabólico $(16,23,33,34)$.
Jeha e cols. evidenciaram baixa sensibilidade do $\mathrm{CGMS}^{\circledR}$ para detecção dos episódios de hipoglicemia (70\%), com predomínio de hipoglicemia assintomática no período noturno e maior eficácia na detecção dos episódios de hiperglicemia pós-prandial em crianças com DMl menores que 6 anos (16). Este achado também foi observado em um estudo brasileiro que avaliou 53 indivíduos, 86,8\% com DMl e idade de 29,74 $\pm 16,38$ anos, submetidos à monitorização contínua da glicose por 72 horas (sensibilidade para hipoglicemia de $75 \%)$, com redução significativa dos níveis da Alc $(8,78 \pm 1,4$ para $7,50 \pm 1,3 ; p=0,001)$, três meses após o exame, boa tolerabilidade e promovendo ajustes na terapêutica (ajuste de dose de insulina, modificação do tipo de insulina utilizada, início de contagem de carboidratos e atividade física) de todos os casos avaliados (35).

Outro estudo brasileiro, dos mesmos autores, mostrou que o $\mathrm{CGMS}^{\circledast}$ foi mais eficaz na detecção de excursões glicêmicas em relação à glicemia capilar $(\mathrm{p}=$ $0,001)$. A hiperglicemia pós-prandial foi evidenciada em 76,9\% $(172,2 \pm 50,8 \mathrm{mg} / \mathrm{dL}, \mathrm{VR}<140 \mathrm{mg} / \mathrm{dL})$, hipoglicemia assintomática detectadas em $58,2 \%$ e o estado de hiper, hipo e normoglicemias foi detectado em $9,7 \% \pm 8,7 \%, 46,2 \% \pm 20,9 \%$ e $43,2 \% \pm 18,6 \%$ dos registros do CGMS ${ }^{\circledR}$ em 72 horas, respectivamente (36). O coeficiente de correlação encontrado entre os valores de glicemia capilar e CGMS $^{\circledR}$, durante o período de hipoglicemia, demonstrou baixa concordância, sem significância estatística $(\mathrm{p}=0,16)$ quando comparado com o período de hiperglicemia $(\mathrm{p}=0,002)$ ou normoglicemia $(\mathrm{p}=0,05)$, encontradas baixa sensibilidade $(79,1 \%)$ e alta especificidade $(97,5 \%)$ para hipoglicemia assintomática quando comparada com episódios de hiper e normoglicemias (36).

Estudo randomizado controlado, envolvendo 109 pacientes de 19 a 76 anos com DM, em uso de insulina, avaliou o efeito do $\mathrm{CGMS}^{\circledast}{ }^{\mathrm{em}}$ relação à automonitorização convencional ao controle glicêmico. Não se observou diferença nos níveis de Alc entre os grupos. Entretanto, o grupo de intervenção $\left(\mathrm{CGMS}^{\circledast}\right)$ apresentou significativa redução na duração das hipoglicemias ao longo de 12 semanas de observação (37). Estudos não controlados iniciais $(31,38)$ e ensaios crossover (29) evidenciaram algum benefício na redução da Alc comparativamente à automonitorização intermitente com a glicemia capilar. Porém, estudos controlados e com maior número de pacientes não foram capazes de reproduzir esse achado $(23,37)$. O CGMS ${ }^{\circledast}$ é desprovido 
de efeitos adversos significativos, embora em até 50\% dos pacientes possam ocorrer discreta irritação local relacionada ao adesivo ou prurido $(34,39,40)$.

Atualmente, existem diversos sensores capazes de realizar a monitorização contínua da glicose, alguns em tempo real $(40,41)$. Esses sistemas têm potencial para revolucionar o tratamento do $\mathrm{DMl}$, pois proporcionam ao paciente informações sobre os perfis glicêmicos pós-prandial e noturno, os quais são raramente obtidos pelo sistema de automonitorização convencional da glicose. Eles também são "intuitivos", pois indicam a direção e o índice de variação glicêmica e são equipados com alarmes para impedir hipoglicemias ou hiperglicemias graves $(40,41)$.

O primeiro aparelho aprovado pela US Food and Drug Administration (FDA) foi o Gluco Watch G2 Biographer ("GlucoWatch", Cygnus, Inc, Redwood City, CA) $(42,43)$. Esse instrumento inicialmente não demonstrou efeito na melhora dos níveis da Alc nem na freqüência de hipoglicemia por ser de difícil manejo, causar irritação na pele, ter acurácia menor que a esperada e não ser usado com a freqüência suficiente para ter impacto no manejo do diabetes (44-46). No entanto, mais recentemente, o aprimoramento desses dispositivos tem aumentado a acurácia e a eficácia desses sensores $(47,48)$.

Os modelos de sistema de monitorização da glicose em tempo real aprovados pela FDA são o Guardian ${ }^{\circledR}$ RT (Minimed, Northridge, CA) e o DexCom ${ }^{\mathrm{TM}}$ STS $^{\mathrm{TM}}$ System (DexCom Inc, San Diego, CA). Fornecem os níveis de glicose continuamente a partir de um pequeno eletrodo inserido no fluido intersticial abaixo da pele. Um transmissor envia a informação através de telemetria para um monitor, sem necessidade de conexão a cabo. Os valores da glicose podem ser visualizados em tempo real no próprio visor do aparelho ou armazenados para posterior transferência e visualização em um computador (49).

A principal vantagem dos aparelhos em tempo real é que permitem que o próprio paciente realize ajustes na terapêutica insulínica (basal ou bolus), de acordo com os resultados obtidos da glicemia de determinado momento, bem como avalie as oscilações glicêmicas ao longo do dia. Porém, preconiza-se também realizar a automonitorização pelo menos três vezes ao dia durante o período da monitorização contínua e trocar o dispositivo a cada três dias $(50)$. De modo diferente, o CGMS $^{\circledR}$ é uma ferramenta no manejo dos pacientes com DM, usado principalmente em consultórios, labo- ratórios ou hospitais, pois os resultados das glicemias somente podem ser visualizados após a transferência dos dados para um software.

A efetividade desses monitores em tempo real depende principalmente de sua acurácia e do fácil manuseio, presente nos dispositivos atuais $(44,45)$. Esses sistemas já têm sido utilizados tanto em adultos quanto em crianças com DM, demonstrando resultados satisfatórios por boa aceitação, fácil manuseio e registro fidedigno dos dados. Eles proporcionam diminuição na variabilidade glicêmica, diminuição nos valores da glicose acima dos níveis-alvo e melhora nos níveis da Alc (39).

Em um estudo realizado com sensor transcutâneo para monitorização contínua de glicose em tempo real, os pacientes utilizaram o sistema durante sete dias consecutivos. Aqueles que apresentavam Alc basal mais elevada (> $10 \%$ ) foram os que mais se beneficiaram com seu uso, adquirindo melhora no controle glicêmico em todos os horários do dia. Além disso, seu uso foi seguro e bem tolerado. Nesse estudo, o desempenho do sensor foi estável durante os sete dias e não demonstrou declínio em sua acurácia durante esse período (51).

O estudo GuardControl demonstrou que, em três meses, com o sistema de monitorização contínua Guardian ${ }^{\circledR}$, foi capaz de proporcionar significativa melhora da Alc em 162 pacientes (adultos e crianças) com DMl mal controlado, apesar da terapia insulínica intensiva. Esses benefícios foram secundários à possibilidade do auto-ajuste de doses de insulina, de acordo com a ingestão alimentar e atividade física (50). A repercussão clínica nos níveis de Alc ou nos resultados a longo prazo ainda são conflitantes. Alguns estudos demonstram que o tempo de uso de um a dois dias por semana por seis meses é curto para causar alguma modificação dos níveis da $\mathrm{Alc}$ (45). Porém, um ensaio clínico randomizado demonstrou redução de, pelo menos, $1 \%$ em $50 \%$ dos pacientes avaliados e de até $2 \%$ em $25 \%$ dos casos com o uso dos sistemas de monitorização em tempo real em pacientes com DMl com mau controle glicêmico prévio $(52)$.

Outro estudo utilizando o $\operatorname{DexCom}^{\mathrm{TM}}$ incluiu 15 pacientes com $\mathrm{DMl}$ que usaram o sensor de duas maneiras: durante 16 dias sem visualização das glicemias e 17 dias permitindo a visualização destas, com ajustes nas doses de insulina de acordo com os resultados glicêmicos e os alarmes de hipo ou hiperglicemias (53). No período em que os pacientes puderam visualizar suas glicemias, foi detectado um tempo $47 \%$ menor com glicemias abai- 
xo de $3,1 \mathrm{mmol} / \mathrm{L}(<56 \mathrm{mg} / \mathrm{dL})$ e $25 \%$ menor com glicemias acima de $13,3 \mathrm{mmol} / \mathrm{L}$ ( $>240 \mathrm{mg} / \mathrm{dL}$ ) quando comparado com o período de não-visualização (53). Evidentemente, investigações futuras se fazem necessárias para mais bem definir recomendações de tratamento com o uso do sistema de monitorização em tempo real, bem como sua eficácia a longo prazo, reprodutibilidade clínica em pacientes com bom controle glicêmico e efeito na incidência de hipoglicemias (54).

Atualmente, estão disponíveis no mercado também os sistemas de monitorização contínua em tempo real integrados com as bombas de insulina (55-57). O MiniMed Paradigm REAL-Time System foi o primeiro a ser lançado, após aprovação da FDA, em abril de 2006. É o mais próximo do que se imagina ser um "pâncreas artificial". O FreeStyle Navigator ("Navigator", Abbott Diabetes Care, Alameda, CA), também lançado recentemente, utiliza um sensor eletroquímico com base em glicose oxidase, que é inserido subcutaneamente e mede a glicose intersticial em intervalos de 20 a 500 $\mathrm{mg} / \mathrm{dL}$, a cada 60 segundos (ou 1.440 leituras ao dia). $\mathrm{O}$ instrumento tem alarmes ajustáveis para hipo e hiperglicemias, e para valores fora das metas de glicemia, permitindo também indicar oscilações glicêmicas (39).

Esses dispositivos têm demonstrado boa acurácia quando comparados com outros métodos de controle glicêmico. A acurácia das medidas da glicose do fluido intersticial feita por meio da monitorização contínua com o FreeStyle Navigator foi comparada com medidas de referência de sangue venoso em 58 pacientes com DMl em um estudo multicêntrico. Os resultados do FreeStyle Navigator foram consistentes e acurados quando comparados com as amostras venosas utilizando método e laboratório de referência (58). O tempo de utilização preconizado é de três dias, mas existem relatos demonstrando segurança e acurácia com maior tempo de uso. Em 20 crianças que utilizaram o sensor por sete dias consecutivos, $89 \%$ dos sensores ainda estavam funcionando após cinco dias, e $78 \%$ funcionaram até o final de sete dias, demonstrando que seu uso pode ser estendido até esse período. A partir do quinto dia, os pacientes referiram menor desconforto com seu uso, porém preferiram utilizar o monitor por, no máximo, cinco a seis dias (40).

A melhora do controle glicêmico com esses aparelhos ainda não está bem estabelecida. Em um estudopiloto, recentemente publicado, que avaliou o uso do sensor acoplado à bomba de infusão contínua de insu- lina (Minimed Paradigm) em dez crianças com DMl, com média de idade de $14,5 \pm 2,6$ anos, evidenciaramse melhora do controle glicêmico e dos níveis de Alc de forma semelhante ao $\mathrm{CGMS}^{\circledR}$, porém em menor tempo, e diminuição dos episódios de hipo e hiperglicemias (57). Esse estudo sugere que a monitorização da glicose em tempo real associada a uma bomba de insulina em crianças com DMl pode ser capaz de evitar complicações crônicas da doença relacionadas às anormalidades glicêmicas (57). No entanto, mais ensaios clínicos randomizados necessitam ser realizados para determinar sua real contribuição e definir a importância desses sensores na diminuição da incidência dos episódios de hipoglicemias, principalmente em pacientes que apresentam bom controle glicêmico.

Existem relatos de alguns efeitos adversos relacionados ao uso desses instrumentos. Podem ocorrer reações de pele por causa do adesivo utilizado para fixar o sensor, mas geralmente são reações leves e de fácil resolução. Também pode ocorrer alguma dificuldade na colocação do sensor em crianças menores em razão de seu tamanho. O Minimed Paradigm foi avaliado quanto ao desempenho, à segurança e à tolerância em 20 crianças com DMl durante sete dias consecutivos. Os resultados demonstraram que os sensores foram bem aceitos, exceto por prurido no local do adesivo, que ocorreu em 29\% dos casos. Em 3\% houve infecção no local do sensor $(40)$.

Muitas empresas estão desenvolvendo sistemas de monitorização não-invasiva, via adesivos ou sensores colocados sobre a pele. Entretanto, esta tecnologia necessita ainda ser refinada. Um modelo que ainda está em estudos nos Estados Unidos é o Glucoband, que é a nova geração do problemático GlucoWatch, uma espécie de relógio de pulso que não necessita penetrar na pele (59).

\section{EXPERIÊNCIA DO INSTITUTO DA CRIANÇA COM DIABETES}

O Instituto da Criança com Diabetes (ICD) disponibiliza para os pacientes com DM em acompanhamento no serviço a colocação de dois sensores de monitorização contínua de glicose $\left(\mathrm{CGMS}^{\circledR}\right)$. Inicialmente, era preconizado o uso do CGMS ${ }^{\circledR}$ por período de três dias, passou-se posteriormente a indicar o exame durante cinco dias e atualmente realiza-se o exame durante sete dias, fazendo-se um download dos resultados no tercei- 
ro dia, para possibilitar o ajuste terapêutico que é reavaliado no período restante do exame.

Foram avaliados retrospectivamente os resultados do CGMS $^{\circledast}$ em 69 indivíduos com DMl, sendo 53,6\% $(37 / 69)$ do sexo masculino. A indicação do CGMS $^{\circledR}$ foi em $66,7 \%(46 / 69)$ por oscilações na glicemia, $17,4 \%$ (12/69) por hipoglicemias, sendo $25 \%$ (3/12) graves e $25 \%(3 / 12)$ noturnas, $8,7 \%$ para otimização de tratamento em indivíduos com infusão contínua de insulina, $2,9 \%$ hiperglicemia e $2,9 \%$ por outras causas. A média de idade na colocação do CGMS $^{\circledR}$ foi de $15,1 \pm 6,4$ anos (2,5 a 40,3 anos) e o tempo médio de duração do diabetes de $7,1 \pm 4,6$ anos $(0,7$ a 21 anos $)$.

O tempo médio de duração do exame foi de $5,2 \pm$ 1,8 dias (três a sete dias); somente em um paciente o exame foi suspenso no primeiro dia de colocação em razão de desconforto. Vinte pacientes $(29,4 \%)$ permaneceram com o CGMS ${ }^{\circledast}$ por período de três dias, seis $(8,8 \%)$ por período de quatro dias, nove $(13,2 \%)$ durante cinco dias e $33(48,6 \%)$ durante período de sete dias.

O local de inserção do sensor foi o abdômen em $94,2 \%$, e $3,8 \%$ os glúteos. A maioria dos pacientes $(88,2 \%)$ não referiu dificuldades na instalação do CGMS $^{\circledR}$, mas em oito pacientes $(11,8 \%)$ foram evidenciados sangramento e dor. Somente 12 pacientes $(17,6 \%)$ referiram prejuízos no desempenho de atividades, como: banho (quatro pacientes), sono (três) e prática de esportes (cinco). Sete pacientes $(10,3 \%)$ necessitaram recolocação do sensor e em 13 pacientes o exame foi interrompido por desinserção ou falha de calibração, antes do tempo previsto.

Trinta e seis pacientes realizaram avaliação parcial dos resultados no terceiro dia e apresentaram melhora no controle glicêmico, observado no período restante do teste. $\mathrm{O} \mathrm{CGMS}^{\circledast}$ foi capaz de promover mudanças e ajustes terapêuticos (basal e/ou bolus, relação carboidrato/insulina, tipos de insulina) em todos os pacientes.

A GC média durante o CGMS $^{\circledR}$ foi de $184,8 \pm 41,1$ $\mathrm{mg} / \mathrm{dL}$, e a média da glicemia no sensor, $183,3 \pm 40,1$ $\mathrm{mg} / \mathrm{dL}$, não havendo diferença significativa entre os valores da glicose captados pelo CGMS ${ }^{\circledR}$ e GC $(p=0,77)$.

Os episódios de hipoglicemias foram detectados em $11,9 \% \pm 10,8 \%$ (mediana: $9 \%, 0 \%$ a $65 \%$ ) do período do exame, $39,7 \% \pm 17,8 \%$ (mediana: $41 \%, 10 \%$ a $68 \%$ ) de glicemias dentro dos limites de normalidade e hiperglicemias $48,3 \pm 14,3 \%$ (mediana: $48 \%, 0 \%$ a $89 \%$ ). O tempo médio de duração de hipoglicemias no período total do exame foi: $11,3 \pm 8,8$ horas (mediana: 11,6 horas). $\mathrm{O}$ número total de excursões glicêmicas foi $19,3 \pm 10,6$, variando de 3,0 a 42,0 (mediana: 10,6), sendo o número total de excursões hiperglicêmicas: $11,9 \pm 6,6$, variando de 0 a 27,0 (mediana: 11,0) e de hipoglicemias: 7,5 \pm 5,0 , variando de 0 a 21,0 (mediana: 7,0).

O controle metabólico na avaliação inicial, $3,6 \mathrm{e}$ 12 meses após o $\mathrm{CGMS}^{\circledR}$, não evidenciou redução dos valores da Alc $[8,7 \% \pm 1,8 \%$ (n: 59$), 9,2 \% \pm 2,0 \%$ (n: 4l) $9,4 \% \pm 2,0 \%(\mathrm{n}: 4 \mathrm{l})$ e $9,5 \% \pm 2,2 \%(\mathrm{n}: 39), \mathrm{p}=$ $0,24]$. No entanto, 19 pacientes mantiveram valores de Alc estáveis $(8,5 \% \pm 1,4 \%$ e $8,4 \% \pm 1,3 \%, \mathrm{p}=0,77), 13$ indivíduos melhoraram (Alc: $10,1 \% \pm 2,3 \%$ e $8,7 \% \pm$ $1,9 \%, \mathrm{p}=0,037)$ e nove apresentaram piora da Alc, porém sem significância estatística (Alc: $9,0 \% \pm 0,7 \% \mathrm{e}$ $10,5 \% \pm 0,8 \%, \mathrm{p}=0,19)$ três meses após o $\mathrm{CGMS}^{\circledR}$.

\section{CONCLUSÃo}

Esta revisão demonstra a eficácia e a acurácia da monitorização contínua da glicose tanto pelo CGMS ${ }^{\circledast}$ quanto em tempo real, na detecção de excursões glicêmicas, episódios de hiperglicemia pós-prandial e hipoglicemias, redução dos níveis de Alc e possibilidade de ajustes terapêuticos em indivíduos que apresentam diabetes melito. Também mostra que o CGMS ${ }^{\circledR}$ é um método seguro, com boa tolerabilidade e capaz de auxiliar na interpretação dos valores da $\mathrm{HbAlc}$ nos pacientes com diabetes melito tipo 1 .

\section{REFERÊNCIAS}

1. The Diabetes Control and Complications Trial Research Group. The effect of intensive treatment of diabetes on the development and progression of long-term complications in insulindependent diabetes mellitus. N Engl J Med. 1993;329:977-86.

2. Epidemiology of Diabetes Interventions and Complications (EDIC). Design, implementation, and preliminary results of a long-term follow-up of the Diabetes Control and Complications Trial cohort. Diabetes Care. 1999;22:99-111.

3. UK Prospective Diabetes Study (UKPDS) Group. Intensive bloodglucose control with sulphonylureas or insulin compared with conventional treatment and risk of complications in patients with type 2 diabetes (UKPDS 33). Lancet. 1998;352:837-53.

4. The American Diabetes Association, European Association for the Study of Diabetes, International Federation of Clinical Chemistry and Laboratory Medicine, and the International Diabetes Federation. Consensus statement on the worldwide standardization of the hemoglobin A1C measurement: Diabetes Care. 2007;30:2399-400.

5. American Association of Clinical Endocrinologists. Medical guidelines for clinical practice for the management of diabetes mellitus. Endocr Pract . 2007;(13 Suppl)1:1-68. 
6. Standards of medical care in diabetes. Diabetes Care. 2006;29(Suppl 1):S4-42.

7. American College of Endocrinology and American Diabetes Association. Consensus statement on inpatient diabetes and glycemic control. Endocr Pract. 2006;12:458-68.

8. European Diabetes Policy Group. A desktop guide to Type 2 diabetes mellitus. Diabet Med. 1999;16:716-30.

9. Guidelines. National Institute of Clinical Excellence. Diabet Med. 2005;22(Suppl 1):5-6.

10. Hainsworth T. NICE guidance on diagnosis and management of type 1 diabetes. Nurs Times. 2004;100:28-9.

11. Jones TW, Davis EA. Hypoglycemia in children with type 1 diabetes: current issues and controversies. Pediatr Diabetes. 2003;4:143-50.

12. Sale MM, Hazelwood K, Zimmet PZ, et al. Trends in diabetes management practices of patients from an Australian insulintreated diabetes register. Diabet Med. 2004;21:165-70.

13. Yates K, Hasnat Milton A, Dear K, Ambler G. Continuous glucose monitoring-guided insulin adjustment in children and adolescents on near-physiological insulin regimens: a randomized controlled trial. Diabetes Care. 2006;29:1512-7.

14. Lagarde WH, Barrows FP, Davenport ML, Kang M, Guess HA, Calikoglu AS. Continuous subcutaneous glucose monitoring in children with type 1 diabetes mellitus: a single-blind, randomized, controlled trial. Pediatr Diabetes. 2006;7:159-64.

15. Guerci B, Benichou M, Floriot M, et al. Accuracy of an electrochemical sensor for measuring capillary blood ketones by fingerstick samples during metabolic deterioration after continuous subcutaneous insulin infusion interruption in type 1 diabetic patients. Diabetes Care. 2003;26:1137-41.

16. Jeha GS, Karaviti LP, Anderson B, et al. Continuous glucose monitoring and the reality of metabolic control in preschool children with type 1 diabetes. Diabetes Care. 2004;27:2881-6.

17. McCall AL, Cox DJ, Crean J, Gloster M, Kovatchev BP. A novel analytical method for assessing glucose variability: using CGMS in type 1 diabetes mellitus. Diabetes Technol Ther. 2006;8:644-53.

18. Zavalkoff SR, Polychronakos C. Evaluation of conventional blood glucose monitoring as an indicator of integrated glucose values using a continuous subcutaneous sensor. Diabetes Care. 2002;25:1603-6.

19. Mastrototaro J. The MiniMed Continuous Glucose Monitoring System (CGMS). J Pediatr Endocrinol Metab. 1999; 3(Suppl 12):751-8.

20. Gross TM, Bode BW, Einhorn D, et al. Performance evaluation of the MiniMed continuous glucose monitoring system during patient home use. Diabetes Technol Ther. 2000;2:49-56.

21. Boyne MS, Silver DM, Kaplan J, Saudek CD. Timing of changes in interstitial and venous blood glucose measured with a continuous subcutaneous glucose sensor. Diabetes. 2003;52:2790-4.

22. Djakoure-Platonoff $C$, Radermercker R, Reach G, Slama G, SeIam JI. Accuracy of the continuous glucose monitoring system in inpatient and outpatient conditions. Diabetes Metab. 2003;29:159-62.

23. Chico A, Vidal-Rios P, Subira M, Novials A. The continuous glucose monitoring system is useful for detecting unrecognized hypoglycemias in patients with type 1 and type 2 diabetes but is not better than frequent capillary glucose measurements for improving metabolic control. Diabetes Care. 2003;26:1153-7.

24. Sachedina N, Pickup JC. Performance assessment of the Medtronic-MiniMed Continuous Glucose Monitoring System and its use for measurement of glycaemic control in Type 1 diabetic subjects. Diabet Med. 2003;20:1012-5.

25. Kaufman FR, Austin J, Neinstein A, et al. Nocturnal hypoglycemia detected with the Continuous Glucose Monitoring System in pediatric patients with type 1 diabetes. J Pediatr. 2002;141:625-30.

26. Schiaffini R, Ciampalini P, Fierabracci $A$, et al. The Continuous Glucose Monitoring System (CGMS) in type 1 diabetic children is the way to reduce hypoglycemic risk. Diabetes Metab Res Rev. 2002;18:324-9.

27. The accuracy of the CGMS in children with type 1 diabetes: results of the diabetes research in children network (DirecNet) accuracy study. Diabetes Technol Ther. 2003;5:781-9.

28. Hay LC, Wilmshurst EG, Fulcher G. Unrecognized hypo- and hyperglycemia in well-controlled patients with type 2 diabetes mellitus: the results of continuous glucose monitoring. Diabetes Technol Ther. 2003;5:19-26.

29. Ludvigsson J, Hanas R. Continuous subcutaneous glucose monitoring improved metabolic control in pediatric patients with type 1 diabetes: a controlled crossover study. Pediatrics. 2003;111:933-8.

30. Schaepelynck-Belicar P, Vague P, Simonin G, Lassmann-Vague $\mathrm{V}$. Improved metabolic control in diabetic adolescents using the continuous glucose monitoring system (CGMS). Diabetes Metab. 2003;29:608-12.

31. Boland E, Monsod T, Delucia M, Brandt CA, Fernando S, Tamborlane WV. Limitations of conventional methods of self-monitoring of blood glucose: lessons learned from 3 days of continuous glucose sensing in pediatric patients with type 1 diabetes. Diabetes Care. 2001;24:1858-62.

32. Guerci B, Floriot M, Bohme $P$, et al. Clinical performance of CGMS in type 1 diabetic patients treated by continuous subcutaneous insulin infusion using insulin analogs. Diabetes Care. 2003;26:582-9.

33. Fiallo-Scharer R. Eight-point glucose testing versus the continuous glucose monitoring system in evaluation of glycemic control in type 1 diabetes. J Clin Endocrinol Metab. 2005;90: 3387-91.

34. Maia FF, Araujo LR [Accuracy, effect on insulin therapy and glycemic control and complications of the continuous glucose monitoring system in type 1 diabetic patients]. Arq Bras Endocrinol Metabol. 2005;49:563-8.

35. Maia FF, Araujo LR [Metabolic/ glycemic control accuracy, effect and complications of the continuous glucose monitoring system in diabetic patients]. Rev Assoc Med Bras 2006;52:395-400.

36. Maia FF, Araujo LR. Efficacy of continuous glucose monitoring system (CGMS) to detect postprandial hyperglycemia and unrecognized hypoglycemia in type 1 diabetic patients. Diabetes Res Clin Pract. 2007;75:30-4.

37. Tanenberg R, Bode B, Lane W, et al. Use of the Continuous Glucose Monitoring System to guide therapy in patients with insulin-treated diabetes: a randomized controlled trial. Mayo Clin Proc. 2004;79:1521-6.

38. Kaufman FR, Gibson LC, Halvorson M, Carpenter S, Fisher LK, Pitukcheewanont $\mathrm{P}$ A pilot study of the continuous glucose monitoring system: clinical decisions and glycemic control after its use in pediatric type 1 diabetic subjects. Diabetes Care. 2001;24:2030-4.

39. Buckingham B, Beck RW, Tamborlane WV, et al. Continuous glucose monitoring in children with type 1 diabetes. J Pediatr. 2007; 151:388-93, 393 e 1-2.

40. Wong LJ, Buckingham BA, Kunselman B, Istoc E, Leach J, Purvis $R$. Extended use of a new continuous glucose monitoring 
system with wireless data transmission in children with type 1 diabetes mellitus. Diabetes Technol Ther. 2006;8:139-45.

41. Updike SJ, Shults MC, Gilligan BJ, Rhodes RK. A subcutaneous glucose sensor with improved longevity, dynamic range, and stability of calibration. Diabetes Care. 2000;23:208-14.

42. Sage BH, Jr. FDA panel approves Cygnus's noninvasive GlucoWatch. Diabetes Technol Ther. 2000;2:115-6.

43. GlucoWatch approved for children with diabetes. FDA Consum. 2002;36:6.

44. Accuracy of the GlucoWatch G2 Biographer and the continuous glucose monitoring system during hypoglycemia: experience of the Diabetes Research in Children Network. Diabetes Care. 2004;27:722-6.

45. lafusco D, Errico MK, Gemma C, Prisco F. Usefulness or uselessness of GlucoWatch in monitoring hypoglycemia in children and adolescents. Pediatrics. 2004;113:175-6; author reply 175-6.

46. Chase HP, Beck R, Tamborlane $\mathrm{W}$, et al. A randomized multicenter trial comparing the GlucoWatch Biographer with standard glucose monitoring in children with type 1 diabetes. Diabetes Care. 2005;28:1101-6.

47. Tansey MJ, Beck RW, Buckingham BA, et al. Accuracy of the modified Continuous Glucose Monitoring System (CGMS) sensor in an outpatient setting: results from a diabetes research in children network (DirecNet) study. Diabetes Technol Ther 2005;7:109-14.

48. Hathout E, Patel N, Southern C, et al. Home use of the GlucoWatch G2 biographer in children with diabetes. Pediatrics. 2005;115:662-6.

49. Iscoe KE, Campbell JE, Jamnik V, Perkins BA, Riddell MC. Efficacy of continuous real-time blood glucose monitoring during and after prolonged high-intensity cycling exercise: spinning with a continuous glucose monitoring system. Diabetes Technol Ther. 2006;8:627-35.

50. Tubiana-Rufi N, Riveline JP, Dardari D. Real-time continuous glucose monitoring using Guardian((R))RT: from research to clinical practice. Diabetes Metab. 2007.

51. Garg S, Zisser H, Schwartz S, et al. Improvement in glycemic excursions with a transcutaneous, real-time continuous glu- cose sensor: a randomized controlled trial. Diabetes Care. 2006;29:44-50.

52. Deiss D, Hartmann R, Hoeffe J, Kordonouri O. Assessment of glycemic control by continuous glucose monitoring system in 50 children with type 1 diabetes starting on insulin pump therapy. Pediatr Diabetes. 2004;5:117-21.

53. Garg SK, Schwartz S, Edelman SV. Improved glucose excursions using an implantable real-time continuous glucose sensor in adults with type 1 diabetes. Diabetes Care. 2004;27:734-8.

54. Deiss D, Bolinder J, Riveline JP, et al. Improved glycemic control in poorly controlled patients with type 1 diabetes using real-time continuous glucose monitoring. Diabetes Care. 2006;29:2730-2.

55. Pohar SL. Subcutaneous open-loop insulin delivery for type 1 diabetes: Paradigm Real-Time System. Issues Emerg Health Technol. 2007;1-6.

56. Mastrototaro JJ, Cooper KW, Soundararajan G, Sanders JB, Shah RV. Clinical experience with an integrated continuous glucose sensor/insulin pump platform: a feasibility study. Adv Ther. 2006;23:725-32.

57. Halvorson M, Carpenter S, Kaiserman K, Kaufman FR. A pilot trial in pediatrics with the sensor-augmented pump: combining real-time continuous glucose monitoring with the insulin pump. J Pediatr. 2007; 150:103-105 e1.

58. Weinstein RL, Schwartz SL, Brazg RL, Bugler JR, Peyser TA, McGarraugh GV. Accuracy of the 5-day FreeStyle Navigator Continuous Glucose Monitoring System: comparison with frequent laboratory reference measurements. Diabetes Care. 2007;30:1125-30.

59. Kordella T. An "ouchless" future? Will the day come when you no longer have to prick, stick, jab or stab to check your glucose levels? Diabetes Forecast. 2005;58:51-53.

Endereço para correspondência:

Balduino Tschiedel

Rua Álvares Cabral, 529

91350-250 Porto Alegre, RS

E-mail: tbalduino@ghc.com.br/http://www.icdrs.org.br 